\title{
MULTIPLICATIVE INVARIANTS AND THE FINITE CO-HOPFIAN PROPERTY
}

\author{
J. J. A. M. HUMPHREYS AND F. E. A. JOHNSON
}

Abstract. A group is said to be finitely co-Hopfian when it contains no proper subgroup of finite index isomorphic to itself. It is known that irreducible lattices in semisimple Lie groups are finitely co-Hopfian. However, it is not clear, and does not appear to be known, whether this property is preserved under direct product. We consider a strengthening of the finite co-Hopfian condition, namely the existence of a non-zero multiplicative invariant, and show that, under mild restrictions, this property is closed with respect to finite direct products. Since it is also closed with respect to commensurability, it follows that lattices in linear semisimple groups of general type are finitely co-Hopfian.

§0. Introduction. A group $G$ is finitely co-Hopfian when it contains no proper subgroup of finite index isomorphic to itself. This condition has a natural geometric significance; if $M$ is a smooth closed connected manifold whose fundamental group $\pi_{1}(M)$ is finitely co-Hopfian, then any smooth mapping $h: M \rightarrow M$ of maximal rank is automatically a diffeomorphism (cf. [8]).

From an algebraic viewpoint, this condition is a weakening of a more familiar notion; recall that a group is said to be co-Hopfian when it contains no proper subgroup (of whatever index, finite or infinite) isomorphic to itself. Co-Hopfian groups are finitely co-Hopfian, but the converse is false, shown by the case of finitely generated non-abelian free groups, which are finitely co-Hopfian but not co-Hopfian.

It is known that an irreducible lattice in a linear semisimple Lie group is finitely co-Hopfian; in the case of a Fuchsian group, this is classical, whilst for an irreducible non-Fuchsian lattice, this follows from Mostow rigidity [9, 10], although, in almost all cases, it had previously been demonstrated by Borel [3]. However, the case of a general semisimple lattice, commensurable with a product of both Fuchsian and Mostow rigid factors, does not seem to have been studied previously, and one objective of this paper is to fill this gap.

An immediate difficulty concerns the extent to which the finite co-Hopfian property is preserved under finite direct products. We introduce a class of finitely generated groups, called quasi-lattices, whose properties weakly approximate the class of lattices in semisimple Lie groups; for the precise definition see $\S 2$. In the case of semisimple lattices we may recall Serre's notion of Euler-Poincaré measure [13]. For quasi-lattices this generalizes to that of a multiplicative invariant on the class of subgroups of finite index. Thus, to a subgroup $H$ of finite index in a quasi-lattice $G$, we may associate a non-zero real number $\mu(H)$ 
depending only upon the isomorphism type of $H$ with the property that if $K$ is a subgroup of index $[H: K]$ in $H$, then

$$
\mu(K)=[H: K] \mu(H) .
$$

Any group admitting such a non-zero multiplicative invariant is automatically finitely co-Hopfian; indeed, any subgroup of finite index is also finitely co-Hopfian. We prove the following.

THEOREM A. If $\Gamma_{1}, \ldots, \Gamma_{n}$ are irreducible quasi-lattices, then $\Gamma_{1} \times$ $\cdots \times \Gamma_{n}$ is also a quasi-lattice; in particular, $\Gamma_{1} \times \cdots \times \Gamma_{n}$ admits a non-zero multiplicative invariant.

From Theorem A, we obtain the following result.

THEOREM B. Let $\Gamma$ be a torsion-free lattice in linear semisimple Lie group with finitely many connected components; then $\Gamma$ is a quasi-lattice. In particular, $\Gamma$ admits a non-zero multiplicative invariant.

As a corollary, we obtain the following result.

COROllary C. Let $\Gamma$ be a lattice in linear semisimple Lie group with finitely many connected components; then $\Gamma$ is finitely co-Hopfian.

This generalizes a result of Borel [3].

The plan of this paper is as follows. In $\S 1$, we give equivalent conditions for the existence of a non-zero multiplicative invariant. In $\$ 2$, we review some basic results on direct products and show that the product structure on a direct product can be deformed only trivially provided that the factors are sufficiently nonabelian. In $\S 3$, we introduce the notion of a quasi-lattice, and prove Theorem $\mathrm{A}$. In $\S \S 4$ and 5, we apply the foregoing theory to the class of semisimple linear lattices, and prove Theorem B and Corollary $\mathrm{C}$.

We point out that the existence of a multiplicative invariant in general is more subtle than familiar examples (for example, the Euler characteristic) might suggest. Thus, it is a consequence of Gottlieb's theorem [5] that groups with nonzero Euler characteristic must have trivial centre; indeed, Rosset's generalization shows that such a group has no non-trivial abelian normal subgroup [12]. This property is not shared with groups admitting non-zero multiplicative invariants; in $\S 6$, we give examples of groups with non-trivial centres which admit a nonzero multiplicative invariant. We conclude, in $\$ 7$, with a brief discussion of the question of multiplicative invariants for more general direct products.

§1. Multiplicative invariants. A group $H$ is said to have the finite index property $\mathcal{F} \mathcal{I}$ when for any subgroups $K, K^{\prime}$ of finite index in $H, K \cong K^{\prime} \Longrightarrow$ $[H: K]=\left[H: K^{\prime}\right]$.

A group homomorphism $\iota: H \rightarrow G$ is a co-finite embedding when $\iota$ is injective and when the index $[G: \iota(H)]$ of $\iota(H)$ in $G$ is finite. We say that $H$ has the finite co-index property $\mathcal{F C}$ when given co-finite embeddings $\iota_{r}$ : $H \rightarrow G_{r}(r=1,2), G_{1} \cong G_{2} \Longrightarrow\left[G_{1}: \iota_{1}(H)\right]=\left[G_{2}: \iota_{2}(H)\right]$. The following is straightforward. 
Proposition 1.1. Let $H^{\prime}$ be a subgroup of finite index in $H$ :

(i) if $H$ has property $\mathcal{F} \mathcal{I}$, then so does $H^{\prime}$;

(ii) if $H^{\prime}$ has property $\mathcal{F C}$, then so does $H$.

Slightly less obvious is the following.

PROPOSITION 1.2. If $H$ has property $\mathcal{F} \mathcal{I}$, then $H$ has property $\mathcal{F C}$.

Proof. Let $\iota_{r}: H \rightarrow G_{r}(r=1,2)$ be co-finite embeddings, and suppose that $\varphi: G_{1} \rightarrow G_{2}$ is an isomorphism. Put $K_{1}=\iota_{1}(H) \cap \varphi^{-1}\left(\iota_{2}(H)\right)$ and $K_{2}=$ $\varphi\left(K_{1}\right)=\varphi\left(\iota_{1}(H)\right) \cap \iota_{2}(H)$. Then $K_{i}$ is a subgroup of finite index in $G_{i}$, and since $\varphi:\left(G_{1}, K_{1}\right) \stackrel{\simeq}{\rightarrow}\left(G_{2}, K_{2}\right)$ is an isomorphism of pairs, then

$$
\left[G_{1}: K_{1}\right]=\left[G_{2}: K_{2}\right] .
$$

However, $K_{r} \subset \iota_{r}(H)$, so that

$$
\left[G_{r}: K_{r}\right]=\left[G_{r}: \iota_{r}(H)\right]\left[\iota_{r}(H): K_{r}\right] .
$$

For $r=1,2, K_{r}^{\prime}=\iota_{r}^{-1}\left(K_{r}\right)$ is a subgroup of finite index in $H$, and since $\iota_{r}:\left(H, K_{r}^{\prime}\right) \rightarrow\left(\iota_{r}(H), K_{r}\right)$ is an isomorphism of pairs, then

$$
\left[H: K_{r}^{\prime}\right]=\left[\iota_{r}(H): K_{r}\right] .
$$

Now $\varphi: K_{1} \rightarrow K_{2}$ is also an isomorphism, so that $K_{1}^{\prime}, K_{2}^{\prime}$ are isomorphic subgroups of finite index in $H$. By hypothesis, $H$ has property $\mathcal{F} \mathcal{I}$, so that

$$
\left[H: K_{1}^{\prime}\right]=\left[H: K_{2}^{\prime}\right] .
$$

The desired conclusion $\left[G_{1}: \iota_{1}(H)\right]=\left[G_{2}: \iota_{2}(H)\right]$ follows from (I)-(IV).

Denote by $\mathcal{F}(H)$ the set of subgroups of finite index in a group $H$. A subset $\Psi$ of $\mathcal{F}(H)$ is said to be cofinal when for each $K \in \mathcal{F}(H)$ there exists $K^{\prime} \in \Psi$ such that $K^{\prime} \subset K$. There is a weak converse to Proposition 1.2.

PROPOSITION 1.3. Let $H$ be a group; then $H$ has property $\mathcal{F} \mathcal{I}$ if and only if $\mathcal{F}(H)$ admits a cofinal subset $\Psi$ such that each $K \in \Psi$ has property $\mathcal{F C}$.

Proof. If $H$ has property $\mathcal{F} \mathcal{I}$ then by Propositions 1.1 and 1.2, each $K \in$ $\mathcal{F}(H)$ has property $\mathcal{F C}$. To establish $(\Longrightarrow)$ we may thus take $\Psi=\mathcal{F}(H)$.

For the implication $(\Longleftarrow)$, suppose that $\Psi$ is a cofinal subset of $\mathcal{F}(H)$ with the property that each $K \in \mathcal{F}(H)$ has property $\mathcal{F C}$. Let $K_{1}, K_{2}$ be subgroups of finite index in $H$ such that $K_{1} \cong K_{2}$. Then $K_{1} \cap K_{2}$ has finite index in $H$, so that we may choose $K \in \Psi$ such that $K \subset K_{1} \cap K_{2}$ and $K$ has property $\mathcal{F C}$. Since $[H: K]=\left[H: K_{r}\right]\left[K_{r}: K\right]$ we have

$$
\left[H: K_{1}\right]\left[K_{1}: K\right]=\left[H: K_{2}\right]\left[K_{2}: K\right] .
$$

However, $K$ has property $\mathcal{F C}$ and $K_{1} \cong K_{2}$, so that $\left[K_{1}: K\right]=\left[K_{2}: K\right]$. It follows from $(*)$ that $\left[H: K_{1}\right]=\left[H: K_{2}\right]$, and $H$ has property $\mathcal{F} \mathcal{I}$. 
Let $\mathcal{C}$ be a class of groups; by a multiplicative invariant on $\mathcal{C}$, we mean a function $\mu: \mathcal{C} \rightarrow \mathbb{R}$ such that, for all $G, H, H^{\prime} \in \mathcal{C}$ :

(i) if $H \cong H^{\prime}$, then $\mu(H)=\mu\left(H^{\prime}\right)$; and

(ii) if $H$ embeds as a subgroup of finite index $d$ in $G$, then $\mu(H)=d \mu(G)$.

The following is clear.

PROPOSITION 1.4. If $\mu: \mathcal{F}(H) \rightarrow \mathbb{R}$ is a multiplicative invariant, then either $\mu(K) \neq 0$ for every $K \in \mathcal{F}(H)$ or $\mu$ is identically zero.

The connection with finitely co-Hopfian groups is given by the following.

PROPOSITION 1.5. Let $G$ be a finitely generated group; if $\mathcal{F}(G)$ admits a non-zero multiplicative invariant, then every subgroup of finite index in $G$ is finitely co-Hopfian.

Proof. If $H \in \mathcal{F}(G)$ and $K$ is a subgroup of index $d>1$ in $H$, then $\mu(K)=$ $d \mu(H)$, so that $\mu(K) \neq \mu(H)$. Hence, $K \not H$.

Abstract groups $G_{1}, G_{2}$ are said to be commensurable, written $G_{1} \sim G_{2}$, when there exists a group $H$, and injections $\iota_{r}: H \rightarrow G_{r}(r=1,2)$, such that $\iota_{r}(H)$ has finite index in $G_{r}$. The commensurability class $\langle G\rangle$ is the collection of groups commensurable with $G$. Although it is apparently only a class, when $G$ is finitely generated, $\langle G\rangle$ is equivalent to the set of objects of a small (countable) category, in which morphisms are co-finite embeddings. So, without loss, we may regard $\langle G\rangle$ as a set. From Propositions 1.2 and 1.4 we obtain the following result.

THEOREM 1.6. Let $H$ be a finitely generated group; then the following statements are equivalent:

(i) $\langle H\rangle$ admits a non-zero multiplicative invariant;

(ii) $\mathcal{F}(H)$ admits a non-zero multiplicative invariant;

(iii) $H$ has property $\mathcal{F} \mathcal{I}$;

(iv) $\mathcal{F}(H)$ has a cofinal subset consisting of groups with property $\mathcal{F C}$.

Proof. We begin by showing that (ii) $\Longleftrightarrow$ (iii). Thus, suppose that $\mu$ : $\mathcal{F}(H) \rightarrow \mathbb{R}$ is a non-zero multiplicative invariant, and that $K_{i}$ is a subgroup of finite index $\delta_{i}$ in $H$ for $i=1$, 2, so that $\mu\left(K_{i}\right)=\delta_{i} \mu(H)$. If $K_{1} \cong K_{2}$, then $\mu\left(K_{1}\right)=\mu\left(K_{2}\right)$, thus $\delta_{1} \mu(H)=\delta_{2} \mu(H)$, and since $\mu(H) \neq 0$, it follows that $\delta_{1}=\delta_{2}$, and $H$ has property $\mathcal{F} \mathcal{I}$. This proves (ii) $\Longrightarrow$ (iii).

Conversely, when $H$ has property $\mathcal{F} \mathcal{I}$ the correspondence $K \mapsto[H: K]$ is a well-defined non-zero multiplicative invariant on $\mathcal{F}(H)$. Thus, (ii) $\Longleftrightarrow$ (iii) as claimed.

By Proposition 1.3, (iii) is equivalent to (iv). Moreover, the set of isomorphism classes in $\mathcal{F}(H)$ is a subset of $\langle H\rangle$ so that (i) $\Longrightarrow$ (ii).

To complete the proof, we must show (ii) $\Longrightarrow$ (i). Thus, suppose that $\mu$ : $\mathcal{F}(H) \rightarrow \mathbb{R}$ is a non-zero multiplicative invariant. When $G$ is commensurable with $H$, there is a group $K$ and finite index embeddings $i: K \rightarrow H, j: K \rightarrow G$; 
we define

$$
v(G)=\frac{[H: i(K)]}{[G: j(K)]} \mu(H) .
$$

The model for this step is the "rational Euler characteristic" of [15].

We must show that $v$ is a well-defined function on $\langle H\rangle$. First observe that, given $K$, this definition is independent of the particular embeddings $i, j$. This is because, since $K$ embeds as a subgroup of finite index in $H, K$ has property $\mathcal{F} \mathcal{I}$ by Proposition 1.1 , and hence also has property $\mathcal{F C}$, by Proposition 1.2. In particular, this formula depends only upon the isomorphism type of $K$, which, without loss, can be assumed to be a subgroup of finite index in $H$.

We now show that the formula is independent of $K$. Let $K_{r}$ be a subgroup of finite index in $H(r=1,2)$, and $j_{r}: K_{r} \rightarrow G$ a co-finite embedding. Put $L=K_{1} \cap K_{2}$, and let $\lambda_{r}: L \rightarrow G$ be the composition of the inclusion $L \subset K_{r}$ with $j_{r}: K_{r} \rightarrow G$; then $\left[G: \lambda_{r}(L)\right]=\left[G: j_{r}\left(K_{r}\right)\right]\left[K_{r}: L\right]$, so that

$$
\frac{[H: L]}{\left[G: \lambda_{r}(L)\right]}=\frac{[H: L]}{\left[G: j_{r}\left(K_{r}\right)\right]\left[K_{r}: L\right]} .
$$

Now $[H: L]=\left[H: K_{r}\right]\left[K_{r}: L\right]$, hence

$$
\frac{[H: L]}{\left[G: \lambda_{r}(L)\right]}=\frac{\left[H: K_{r}\right]}{\left[G: j_{r}\left(K_{r}\right)\right]} .
$$

However, again, by Propositions 1.1 and $1.2, L$ has property $\mathcal{F C}$, so that $\left[G: \lambda_{1}(L)\right]=\left[G: \lambda_{2}(L)\right]$, and so

$$
\frac{\left[H: K_{1}\right]}{\left[G: j_{1}\left(K_{1}\right)\right]}=\frac{\left[H: K_{2}\right]}{\left[G: j_{2}\left(K_{2}\right)\right]}
$$

as required. Thus, (ii) $\Longleftrightarrow$ (i), completing the proof.

We say that $H$ admits a non-zero multiplicative invariant when any of the above conditions are fulfilled.

\$2. Rigidity of products. An infinite group $G$ is said to be reducible when it is commensurable with a direct product $G \sim H_{1} \times H_{2}$ of infinite groups $H_{1}, H_{2}$; otherwise, $G$ is irreducible. We say that a group $\Gamma$ has property $\Pi$ when given an expression $\Gamma=\Gamma_{1} \Gamma_{2} \cdots \Gamma_{m}(m \geq 2)$ as a product (not necessarily direct) of mutually centralising normal subgroups $\Gamma_{i}$, then for some $i, \Gamma_{j}=\{1\}$ for $j \neq i$.

PROPOSITION 2.1. Let $\Gamma$ be an infinite irreducible group with trivial centre. If $\Gamma$ has no non-trivial finite normal subgroup, then $\Gamma$ has property $\Pi$.

Proof. Let $\Gamma=\Gamma_{1} \Gamma_{2} \cdots \Gamma_{m}$ be an expression of $\Gamma$ as a product of mutually centralising normal subgroups $(m \geq 2)$. Then some $\Gamma_{i}$ is infinite. Put $\Psi=$ $\Gamma_{1} \cdots \Gamma_{i-1} \Gamma_{i+1} \cdots \Gamma_{m}$. Since the $\Gamma_{j}$ are mutually centralising, $\Gamma_{i} \cap \Psi$ is central in $\Gamma$. Thus, $\Gamma_{i} \cap \Psi=\{1\}$, and $\Gamma \cong \Gamma_{i} \times \Psi$. Since $\Gamma$ is irreducible and $\Gamma_{i}$ is infinite, $\Psi$ is a finite normal subgroup of $\Gamma$, and hence is trivial. Thus, $\Gamma_{j}=\{1\}$ for $j \neq i$, verifying property $\Pi$. 
If $\mathcal{C}$ is a class of groups, then by a $\mathcal{C}$-product structure on a group $G$ we mean a finite sequence $\mathcal{P}=\left(G_{\lambda}\right)_{\lambda \in \Lambda}$ where each $G_{\lambda} \in \mathcal{C}$ is a non-trivial normal subgroup of $G$ such that $G$ is the internal direct product

$$
G=\prod_{\lambda \in \Lambda} G_{\lambda}=G_{\lambda_{1}} \circ \cdots \circ G_{\lambda_{n}},
$$

where $\Lambda=\left\{\lambda_{1}, \ldots, \lambda_{n}\right\}$. Two $\mathcal{C}$-product structures $\mathcal{P}=\left(G_{\lambda}\right)_{\lambda \in \Lambda}, \quad \mathcal{Q}=$ $\left(H_{\omega}\right)_{\omega \in \Omega}$ on $G$ are said to be equivalent when there exists a bijection $\sigma: \Lambda \rightarrow \Omega$ such that for all $\lambda \in \Lambda, G_{\lambda} \cong H_{\sigma(\lambda)}$, and strongly equivalent when, in addition, $G_{\lambda}=H_{\sigma(\lambda)}$ for all $\lambda \in \Lambda$.

Let $\mathcal{Q}$ denote the class of finitely generated torsion-free infinite groups in which every subgroup of finite index has trivial centre. Let $\mathcal{Q}_{0}$ denote the subclass of $\mathcal{Q}$ consisting of irreducible groups. The following can, mutatis mutandis, be proved along the lines of [7, Proposition 6.2].

PROPOSITION 2.2. Let $\Delta$ be a subgroup of finite index in $\Gamma$, and suppose that $\Gamma, \Delta$ both admit $\mathcal{Q}_{0}$-product structures; thus, $\Gamma=\Gamma_{1} \circ \cdots \circ \Gamma_{m}, \Delta=$ $\Delta_{1} \circ \cdots \circ \Delta_{\mu}$, then:

(i) $\quad \mu=m$; and

(ii) there is a unique permutation $\sigma \in \Sigma_{m}$ such that $\Delta_{i} \subset \Gamma_{\sigma(i)}$ for each $i$; in particular

(iii) $\Delta_{i} \cap \Gamma_{j}=\{1\}$ if $j \neq \sigma(i)$, and $\Delta_{i}$ has finite index in $\Gamma_{\sigma(i)}$.

In similar fashion, we also obtain the following.

Proposition 2.3. Let $\Delta, \Delta^{\prime}$ be subgroups of finite index in a group $G$. If $\Delta=\Delta_{1} \circ \cdots \circ \Delta_{m}$ and $\Delta^{\prime}=\Delta_{1}^{\prime} \circ \cdots \circ \Delta_{\mu}^{\prime}$ are $\mathcal{Q}_{0}$-product structures, then:

(i) $\quad \mu=m$; and

(ii) for some unique permutation $\sigma \in \Sigma_{m}, \Delta_{i} \cap \Delta_{\sigma(i)}^{\prime}$ has finite index in each of $\Delta_{i}, \Delta_{\sigma(i)}^{\prime}$, and $\Delta_{i} \cap \Delta_{j}^{\prime}=\{1\}$ if $j \neq \sigma(i)$.

As a consequence, we have the following.

COROLLARY 2.7. Any two $\mathcal{Q}_{0}$-product structures on a group are strongly equivalent.

§3. Quasi-lattices and the product theorem. By a quasi-lattice $G$ we mean a finitely generated $\mathcal{Q}$-group $G$ which admits a non-zero multiplicative invariant. We prove that a finite product of irreducible quasi-lattices is a quasi-lattice.

We first treat a special case; say that a group $\Delta$ is a product of restricted type when $\Delta \cong \Delta_{1} \times \cdots \times \Delta_{N}$, where $\Delta_{1}, \ldots, \Delta_{N}$ are irreducible quasi-lattices such that

$$
\text { for all } i, j, \Delta_{i} \sim \Delta_{j} \Longrightarrow \Delta_{i} \cong \Delta_{j}
$$

Collecting together isomorphic factors, a product $\Delta$ of restricted type may be described in the form $\Delta=\Delta(1) \circ \cdots \circ \Delta(m)$ where for each $1 \leq r \leq m, \Delta(r)=$ $\Delta_{1}(r) \circ \cdots \circ \Delta_{e_{r}}(r)$ where $\Delta_{1}(r), \ldots, \Delta_{e_{r}}(r)$ are irreducible quasi-lattices of 
a single isomorphism type $\Delta_{1}(r) \cong \Delta_{2}(r) \cong \ldots \cong \Delta_{e_{r}}(r)$, and where distinct isomorphism types are pairwise incommensurable. Clearly $N=e_{1}+\cdots+e_{m}$.

A permutation $\sigma$ of $\{1, \ldots, N\}$ which preserves the partition

$$
\begin{aligned}
& \left\{1, \ldots, e_{1}\right\} \coprod\left\{e_{1}+1, \ldots, e_{1}+e_{2}\right\} \\
& \quad \times \coprod \ldots \coprod\left\{e_{1}+\cdots+e_{m-1}+1, \ldots, N\right\}
\end{aligned}
$$

will be written in the form $\sigma=\sigma_{1} \bigsqcup \cdots \amalg \sigma_{m}$ where $\sigma_{r}$ is a permutation of $\left\{1, \ldots, e_{r}\right\}$; that is, we perform a psychological normalization and regard each component of the partition as indexed independently. We note for reference that the indexing now obeys the rule.

(3.2): If $\Delta$ is a product of restricted type, then for any $i, j, r, s$,

$$
\Delta_{i}(r) \sim \Delta_{j}(s) \Longrightarrow r=s \quad \text { and } \quad i, j \in\left\{1, \ldots, e_{r}\right\}
$$

PROPOSITION 3.3. Any product of quasi-lattices of restricted type has property $\mathcal{F C}$.

Proof. Let $\Delta \cong \Delta_{1} \times \cdots \times \Delta_{N}$, be a product of restricted type, and let $\iota_{r}: \Delta \rightarrow \Gamma(r)$ be co-finite embeddings $(r=1,2)$. It should cause no confusion to suppress the symbols $\iota_{r}$ and simply write $\Delta \subset \Gamma(r)$.

Let $\varphi: \Gamma(1) \rightarrow \Gamma(2)$ be an isomorphism, and put $\Delta_{i}^{\prime}(r)=\varphi^{-1}\left(\Delta_{i}(r)\right)$, so that $\Delta^{\prime}(r)$ also has a $\mathcal{Q}_{0}$ product structure $\Delta^{\prime}(r)=\Delta_{1}^{\prime}(r) \circ \cdots \circ \Delta_{e_{r}}^{\prime}(r)$. Putting $\Delta^{\prime}=\varphi^{-1}(\Delta)$ we see also that $\Delta^{\prime}=\Delta^{\prime}(1) \times \cdots \times \Delta^{\prime}(m)$. Observe that $\Delta \cap \Delta^{\prime}$ has finite index in $\Gamma(1)$. The permutation $\sigma$ of $\{1, \ldots, N\}$ provided by Proposition 2.3 must preserve the partition

$$
\begin{gathered}
\left\{1, \ldots, e_{1}\right\} \coprod\left\{e_{1}+1, \ldots, e_{1}+e_{2}\right\} \coprod \ldots \\
\ldots \coprod\left\{e_{1}+\cdots+e_{m-1}+1, \ldots, N\right\}
\end{gathered}
$$

that is, there are permutations $\sigma_{r}$ of $\left\{1, \ldots, e_{r}\right\}$ such that $\Delta_{i}(r)$ intersects $\Delta_{\sigma_{r}(i)}^{\prime}(r)$ in a subgroup of finite index. Put $\Omega_{i}(r)=\Delta_{i}(r) \cap \Delta_{\sigma_{r}(i)}^{\prime}(r)$.

For all $r, j, \Delta_{j}^{\prime}(r)=\varphi^{-1}\left(\Delta_{j}(r)\right)$ is isomorphic to $\Delta_{j}(r)$, and since each $\Delta_{j}(r)$ admits a non-zero multiplicative invariant, then

$$
\left[\Delta_{i}(r): \Omega_{i}(r)\right]=\left[\Delta_{\sigma_{r}(i)}^{\prime}(r): \Omega_{i}(r)\right]
$$

Put $\Omega(r)=\Omega_{1}(r) \circ \cdots \circ \Omega_{m}(r)$. From the identities

$$
[\Delta(r): \Omega(r)]=\prod_{i}\left[\Delta_{i}(r): \Omega_{i}(r)\right]
$$

and

$$
\left[\Delta^{\prime}(r): \Omega(r)\right]=\prod_{i}\left[\Delta_{\sigma_{r}(i)}^{\prime}(r): \Omega_{i}(r)\right]
$$

it follows that $[\Delta(r): \Omega(r)]=\left[\Delta^{\prime}(r): \Omega(r)\right]$. Since $\Delta=\Delta(1) \circ \cdots \circ \Delta(m)$, $\Delta^{\prime}=\Delta^{\prime}(1) \circ \cdots \circ \Delta^{\prime}(m)$ and $\Omega=\Omega(1) \circ \cdots \circ \Omega(m)$ then $[\Delta: \Omega]=\left[\Delta^{\prime}: \Omega\right]$. 
Now $\varphi(\Omega) \subset \Delta$, and $\varphi:\left(\Delta^{\prime}, \Omega\right) \stackrel{\simeq}{\rightarrow}(\Delta, \varphi(\Omega))$ is an isomorphism of pairs; thus, $\left[\Delta^{\prime}: \Omega\right]=[\Delta: \varphi(\Omega)]$, and $[\Delta: \Omega]=[\Delta: \varphi(\Omega)]$. Again, there is an isomorphism of pairs $\varphi:\left(\Gamma_{1}, \Omega\right) \stackrel{\widetilde{\Xi}}{\rightarrow}\left(\Gamma_{2}, \varphi(\Omega)\right)$ so that $\left[\Gamma_{1}: \Omega\right]=\left[\Gamma_{2}\right.$ : $\varphi(\Omega)]$. However, $\left[\Gamma_{1}: \Omega\right]=\left[\Gamma_{1}: \Delta\right][\Delta: \Omega]$ and $\left[\Gamma_{2}: \varphi(\Omega)\right]=\left[\Gamma_{2}: \Delta\right][\Delta:$ $\varphi(\Omega)]$. Hence, $\left[\Gamma_{1}: \Delta\right]=\left[\Gamma_{2}: \Delta\right]$, as claimed.

If $\Delta_{1}, \ldots, \Delta_{m}$ are groups such that $\Delta_{i} \sim \Delta_{j}$ for each $i, j$, then each $\Delta_{i}$ contains a subgroup $\Lambda_{i}$ of finite index such that $\Lambda_{1} \cong \Lambda_{2} \cong \cdots \cong \Lambda_{m}$. Hence, a group $\Delta$ possessing a $\mathcal{Q}_{0}$-product structure $\Delta=\Delta_{1} \circ \cdots \circ \Delta_{m}$ has a subgroup $\Lambda$ of finite index of the form $\Lambda_{1} \circ \cdots \circ \Lambda_{m}$ where (i) $\Lambda_{i} \subset \Delta_{i}$, and (ii) $\Lambda_{i} \sim \Lambda_{j} \Rightarrow \Lambda_{i} \cong \Lambda_{j}$. The next result follows easily.

PROPOSITION 3.4. If $\Gamma$ is a group possessing a subgroup $\Delta$ of finite index which admits a $\mathcal{Q}_{0}$-product structure, then the set of subgroups of finite index in $\Delta$ which are products of restricted type is cofinal in $\mathcal{F}(\Gamma)$.

Now suppose that $\Gamma=\Gamma_{1} \times \cdots \times \Gamma_{N}$ where $\Gamma_{1}, \ldots, \Gamma_{N}$ are irreducible quasi-lattices. It follows from Propositions 3.3 and 3.4 that $\mathcal{F}(\Gamma)$ contains a cofinal subset of groups of type $\mathcal{F C}$. Hence, $\Gamma$ admits a non-zero multiplicative invariant by Theorem 1.6, and we arrive at the following result.

THEOREM A. If $\Gamma_{1}, \ldots, \Gamma_{n}$ are irreducible quasi-lattices, then $\Gamma_{1} \times$ $\cdots \times \Gamma_{n}$ is also a quasi-lattice.

§4. Multiplicative invariants on irreducible semisimple lattices. In this section, we consider examples of multiplicative invariants which arise in practice.

(I) The Euler characteristic.. Let $\Gamma$ be a group of type FP [4]; $\Gamma$ is then finitely generated and has finite cohomological dimension; in particular, $\Gamma$ is torsion free. The Euler characteristic $\chi(\Gamma)$ is then defined in the usual way, and is an integer.

PROPOSITION 4.1. Let $\Gamma$ be a group of type FP. If $\chi(\Gamma) \neq 0$, then $\Gamma$ is a quasi-lattice.

Proof. If $\Delta$ is a subgroup of finite index in $\Gamma$, then $\Delta$ is also of type FP and $\chi(\Delta) \neq 0$, so that $\chi$ gives the required non-zero multiplicative invariant. Moreover, $\Delta$ has trivial centre, by the theorem of Gottlieb [5].

(II) The invariant volume on a rigid semisimple lattice.. Denote by $\mathcal{L}$ the class of infinite linear semisimple lattices; that is, $\Gamma \in \mathcal{L}$ when there exists a noncompact linear Lie group $G$ with finitely many connected components whose identity component $G_{0}$ is semisimple such that $\Gamma$ admits an embedding $\Gamma \subset G$ as a discrete subgroup of $G$, and such that $G / \Gamma$ has finite invariant volume.

In the case where $G$ is a direct product of simple non-compact Lie groups $G=G_{1} \times \cdots \times G_{m}, \Gamma$ is said to be lattice irreducible in $G$ when $\pi_{I}(\Gamma)$ is nondiscrete for every proper non-empty subset $I$ of $\{1, \ldots, m\}$ where

$$
\pi_{I}: G \rightarrow \prod_{i \in I} G_{i}=G_{I}
$$


is the projection. An argument using Borel's density theorem (see [11, p. 86]) shows that when $\Gamma$ is lattice irreducible, it is irreducible in the former sense, namely that it is not commensurable with a direct product of infinite groups. Denote by $\mathcal{L}_{0}$ the subclass of torsion-free irreducible lattices in this sense. If $\Gamma \in \mathcal{L}_{0}$ and $G$ is a connected adjoint semisimple Lie group which contains $\Gamma$ as a discrete subgroup of finite covolume, then $\Gamma$ is said to be a rigid lattice when $(G, \Gamma)$ satisfies Mostow rigidity; that is, any automorphism of $\Gamma$ has a unique extension to $G$. In this case, make a specific choice for the infinitesimal volume element on the tangent space $T G_{\mathrm{Id}}$; when $\Delta \subset \Gamma$ is a subgroup of finite index $d$, define

$$
\mu(\Delta)=\operatorname{vol}(G / \Delta) .
$$

Then $\mu$ is a non-zero multiplicative invariant, since by Mostow rigidity, $\operatorname{vol}(G / \Delta)$ depends only upon the isomorphism class of $\Delta$; that is, we have the following result.

(4.3) A rigid lattice admits a non-zero multiplicative invariant.

§5. Multiplicative invariants for general semisimple lattices. We begin by making the following observation.

\section{Proposition 5.1. If $\Gamma \in \mathcal{L}_{0}$, then $\Gamma$ is an irreducible quasi-lattice.}

Proof. As is well known (cf. [9]), any semisimple linear lattice $\Gamma$ is finitely generated. If $\Gamma$ is torsion free, then by a standard Borel density argument, all of its subgroups of finite index have trivial centre. If $\Gamma$ is a lattice in $\mathrm{PSL}_{2}(\mathbb{R})$, it is either a free non-abelian group of finite rank or the fundamental group of an orientable surface of genus at least two. Either way, $\Gamma$ has type FP, and we take the multiplicative invariant to be the Euler characteristic, which is necessarily non-zero in either case.

When $\Gamma$ is an irreducible non-Fuchsian lattice, $\Gamma$ is Mostow rigid and we take as multiplicative invariant the invariant volume of (4.3).

We now show that Proposition 5.1 remains true without the restriction of irreducibility. We recall briefly the principal structural facts about lattice groups. As observed previously, semisimple linear lattices are finitely generated. Selberg's theorem [2] now shows that a semisimple linear lattice admits a normal subgroup of finite index which is torsion free.

Suppose that $\Gamma \in \mathcal{L}$ is torsion free and embeds as a discrete subgroup of finite covolume in a connected semisimple Lie group; on taking the quotient first by the finite centre of the containing Lie group, and then by its maximal compact normal subgroup, we see that $\Gamma$ embeds as a discrete subgroup of finite covolume in a non-compact connected semisimple adjoint Lie group $G$ which has no non-trivial compact normal subgroup. Thus, $\Gamma$ contains a torsionfree normal subgroup $\Delta$ of finite index such that $\Delta$ embeds as a lattice in a connected non-compact semisimple Lie group. A Borel density argument [11, Theorem 5.22, p. 86] shows that $\Delta$ contains a torsion-free subgroup $\Gamma_{0}$ which admits an $\mathcal{L}_{0}$-product structure, and which has finite index in $\Delta$. A standard argument, conjugating by the finite number of generators and taking 
intersections, allows us, by passing to a further subgroup of finite index if necessary, to assume that $\Gamma_{0}$ is also normal in $\Gamma$. On writing the $\mathcal{L}$-product structure as $\Gamma_{0}=\Gamma_{1} \circ \cdots \circ \Gamma_{m}$, we obtain the following normal form for linear semisimple lattices.

Proposition 5.2. Let $\Gamma \in \mathcal{L}$; then $\Gamma$ occurs in an exact sequence

$$
1 \rightarrow \Gamma_{1} \circ \cdots \circ \Gamma_{m} \rightarrow \Gamma \rightarrow \Phi \rightarrow 1
$$

where $\Gamma_{1}, \ldots, \Gamma_{m} \in \mathcal{L}_{0}$ and $\Phi$ is finite.

From Propositions 5.1 and 5.2 and Theorem A, we now obtain the following.

THEOREM B. Let $\Gamma$ be a torsion-free lattice in a linear semisimple Lie group with finitely many connected components; then $\Gamma$ is a quasi-lattice. In particular, $\langle\Gamma\rangle$ admits a non-zero multiplicative invariant.

As a corollary, we obtain the desired generalization of Borel's result [3].

COROllary C. Let $\Gamma$ be a lattice in linear semisimple Lie group with finitely many connected components; then $\Gamma$ is finitely co-Hopfian.

§6. Non-degenerate central extensions. The examples of finitely co-Hopfian groups considered so far all have trivial centres. However, as we now show, that is not an essential feature, and we give a sufficient condition for a central extension to be finitely co-Hopfian. Let

$$
\mathcal{E}=(0 \rightarrow \mathcal{Z} \rightarrow G \rightarrow \Gamma \rightarrow 1)
$$

be a central extension where $\Gamma$ is a finitely generated group with trivial centre, and $\mathcal{Z}$ is a finitely generated abelian group. The homology spectral sequence of the extension takes the form:

$$
E_{p, q}^{2}=H_{p}\left(\Gamma ; H_{q}(\mathcal{Z} ; \mathbb{Z})\right) \Longrightarrow H_{p+q}(G ; \mathbb{Z})
$$

say that $\mathcal{E}$ is non-degenerate when $\operatorname{Coker}\left(d_{2,0}^{2}: H_{2}(\Gamma ; \mathbb{Z}) \rightarrow H_{1}(\mathcal{Z} ; \mathbb{Z})\right)$ is finite.

THEOREM 6.1. Let $\mathcal{E}=(0 \rightarrow \mathcal{Z} \rightarrow G \stackrel{\pi}{\rightarrow} \Gamma \rightarrow 1)$ be a central extension where $\mathcal{Z}$ is a finitely generated abelian group, and $\Gamma$ is a finitely generated group in which every subgroup of finite index has trivial centre. If $\Gamma$ is finitely co-Hopfian and $\mathcal{E}$ is non-degenerate, then $G$ is finitely co-Hopfian.

Proof. Let $H$ be a subgroup of finite index in $G$. Then $\Delta=H /(H \cap \mathcal{Z})$ is a subgroup of finite index in $\Gamma=G / \mathcal{Z}$. Denote by $\mathcal{Z}_{K}$ the centre of a group $K$. Since $\mathcal{Z}_{\Gamma}$ is trivial, it is clear that $\mathcal{Z}=\mathcal{Z}_{G}$, and the subgroup condition on $\Gamma$ implies that $H \cap \mathcal{Z}=\mathcal{Z}_{H}$.

If $H \cong G$, then $\Delta=H / \mathcal{Z}_{H} \cong G / \mathcal{Z}_{G}=\Gamma$. Since $\Gamma$ is finitely co-Hopfian, it follows that $\Delta=\Gamma$. Thus, without loss of generality, we may suppose that $H$ is defined by an extension $\mathcal{E}^{\prime}=(0 \rightarrow H \cap \mathcal{Z} \rightarrow H \stackrel{\pi}{\rightarrow} \Gamma \rightarrow 1)$. To establish the 
desired conclusion, it suffices to show that $H \cap \mathcal{Z}=\mathcal{Z}$. Denote the homology transgression of the extension $\mathcal{E}^{\prime}$ by

$$
\delta_{2,0}^{2}: H_{2}(\Gamma ; \mathbb{Z}) \rightarrow H_{1}(H \cap \mathcal{Z} ; \mathbb{Z}) .
$$

Let $\iota: H \cap \mathcal{Z} \subset \mathcal{Z}$ denote the inclusion, which is an embedding of finite index $j$, say. There are extensions

$$
\begin{aligned}
& 0 \rightarrow \operatorname{Coker}\left(d_{2,0}^{2}\right) \rightarrow \operatorname{Tor}_{1}\left(H_{1}(G ; \mathbb{Z})\right) \rightarrow \operatorname{Tor}_{1}\left(H_{1}(\Gamma ; \mathbb{Z})\right) \rightarrow 0 \\
& 0 \rightarrow \operatorname{Coker}\left(\delta_{2,0}^{2}\right) \rightarrow \operatorname{Tor}_{1}\left(H_{1}(H ; \mathbb{Z})\right) \rightarrow \operatorname{Tor}_{1}\left(H_{1}(\Gamma ; \mathbb{Z})\right) \rightarrow 0
\end{aligned}
$$

and $\iota$ induces an embedding $\operatorname{Coker}\left(\delta_{2,0}^{2}\right) \rightarrow \operatorname{Coker}\left(d_{2,0}^{2}\right)$ with index $j$. Since $H \cong G$, it follows that $\operatorname{Tor}_{1}\left(H_{1}(G ; \mathbb{Z})\right) \cong \operatorname{Tor}_{1}\left(H_{1}(H ; \mathbb{Z})\right)$ and $\left|\operatorname{Coker}\left(\delta_{2,0}^{2}\right)\right|=$ $\left|\operatorname{Coker}\left(d_{2,0}^{2}\right)\right|$. Hence, $j=1$, so that $H \cap \mathcal{Z}=\mathcal{Z}$ and $H=G$.

Let $\Sigma^{g}$ denote the orientable surface of genus $g$, and let $\Sigma_{g}=\pi_{1}\left(\Sigma^{g}\right)$. Central extensions

$$
\mathcal{E}=\left(0 \rightarrow \mathbb{Z} \rightarrow G \rightarrow \Sigma_{g} \rightarrow 1\right)
$$

are classified up to congruence by a cohomology class $c(\mathcal{E}) \in H^{2}\left(\Sigma_{g} ; \mathbb{Z}\right) \cong \mathbb{Z}$. Denote by $\Sigma(g, r)$ the group defined by the extension

$$
\mathcal{E}(g, r)=\left(0 \rightarrow \mathbb{Z} \rightarrow \Sigma(g, r) \rightarrow \Sigma_{g} \rightarrow 1\right)
$$

with $c(\mathcal{E})=r$. These groups have a geometrical description, namely that $\Sigma(g, r)=\pi_{1}(E(g, r))$, where $E(g, r)$ is the total space of the $S^{1}$-bundle over $\Sigma^{g}$ with Chern class $c_{1}=r$. Analysis of the proof of Theorem 6.1 reveals that a stronger statement is true.

COROLLARY 6.2. When $g \geq 2$ and $r \neq 0$, a subgroup $H$ of finite index $\delta$ takes the form $H \cong \Sigma(h, s)$ where for some positive integers $\delta_{B}, \delta_{F}$ such that $\delta=\delta_{B} \delta_{F}$,

$$
\text { (i) } h=1+\delta_{B}(g-1) \quad \text { and } \quad \text { (ii) } s=\frac{r}{\delta_{F}} \text {. }
$$

If $g \geq 2$ and $r \neq 0$, and $H$ is a subgroup of finite index $\delta$, then $\delta$ is automatically determined by the isomorphism type of $H$; if $H \cong \Sigma(h, s)$, then put

$$
\mu_{g, r}(H)=\frac{r}{s}\left(\frac{h-1}{g-1}\right) \quad(=\delta) .
$$

COROllary 6.3. For $g \geq 2$ and $r \neq 0, \mu_{g, r}$ determines a non-zero multiplicative invariant on $\mathcal{F}(\Sigma(g, r))$.

The groups $\Sigma(g, r)$ embed as lattices in the nonlinear simple group $\widetilde{\mathrm{SL}_{2}(\mathbb{R})}$, the universal covering group of $\mathrm{SL}_{2}(\mathbb{R})$. 
§7. More general direct products. Theorem A gives conclusions which seem difficult to establish more directly; for example, we have the following result.

THEOREM 7.1. Let $\Gamma$ be a lattice in a linear semisimple Lie group with finitely many connected components and let $\Delta$ be a group of type FP with $\chi(\Delta) \neq 0$. Then $\Gamma \times \Delta$ admits a non-zero multiplicative invariant.

Proof. First observe that the normal form theorem for semisimple linear lattices, Proposition 5.2, has an analogue for groups of type FP. Thus, if $\Delta$ is reducible, it splits, up to commensurability, as a product of infinite groups; it is known, [4], that the factors are also of type FP. If the factors are reducible, proceed to split these in the same way. However, the cohomological dimension of $\Delta$ places, a priori, a bound on the number of times splitting may occur. Hence, $\Delta$ is commensurable with a direct product $\Delta_{1} \times \cdots \times \Delta_{n}$ of irreducible groups of type FP. Moreover, since $\chi(\Delta) \neq 0$, then each $\chi\left(\Delta_{i}\right) \neq 0$, and, as in Proposition 4.1, $\Delta_{i}$ is a quasi-lattice. Applying Proposition 5.2 to $\Gamma$, we see that $\Gamma \times \Delta$ is commensurable with a direct product $\Gamma_{1} \times \cdots \times \Gamma_{m} \times \Delta_{1} \times$ $\cdots \times \Delta_{n}$, where each $\Gamma_{i}, \Delta_{j}$ is an irreducible quasi-lattice. The conclusion now follows from Theorem A.

There is an obvious question, as follows.

(*) Does every finitely co-Hopfian group admit a non-zero multiplicative invariant?

One suspects that the answer is "no", but the situation is not clear. For example, the groups $\Sigma(g, r)$ of $\S 6$ are irreducible when $g \geq 2$ and $r \neq 0$. However, since $\Sigma(g, r)$ has non-trivial centre, the argument of Theorem $\mathrm{A}$ does not apply to a product $G=\Sigma\left(g_{1}, r_{1}\right) \times \cdots \times \Sigma\left(g_{m}, r_{m}\right)$, and it is not immediately apparent whether such a product admits a non-zero multiplicative invariant. However, $G$ is still finitely co-Hopfian, as it may be written as an extension

$$
0 \rightarrow \mathbb{Z}^{m} \rightarrow G \stackrel{\pi}{\rightarrow} \Sigma_{g_{1}} \times \cdots \times \Sigma_{g_{m}} \rightarrow 1
$$

satisfying the hypotheses of Theorem 6.1 above. Furthermore, $G$ satisfies Poincaré duality and so by Strebel's theorem [14] is not isomorphic to any subgroup of infinite index; thus, we have the following result.

PROPOSITION 7.2. The groups $\Sigma\left(g_{1}, r_{1}\right) \times \cdots \times \Sigma\left(g_{m}, r_{m}\right)$ are coHopfian provided that for each $i, g_{i} \geq 2$ and $r_{i} \neq 0$.

Free products form another class of examples for which the situation is not entirely clear. Using the theorems of Grushko and Kurosh [6], it is straightforward to show that with the sole exception of $C_{2} * C_{2}$, any finitely generated non-trivial free product $G_{1} * \cdots * G_{k}$ is finitely co-Hopfian. However, the theorem of Baumslag-Dyer-Heller [1] allows us to construct free products of type FP with $\chi=0$, removing the possibility of using the most obvious multiplicative invariant. For example, if $G(n)$ is a Baumslag-DyerHeller group corresponding to the $n$-sphere $S^{n}$ and $F_{2}$ is the free group of rank two, then $\Gamma_{n}=G(2 n) * F_{2}$ is a group of type FP with $\chi\left(\Gamma_{n}\right)=0$. Here $\Gamma_{n}$ 
is torsion free, and every subgroup of finite index, being a free product not isomorphic to $C_{2} * C_{2}$, has trivial centre and is finitely co-Hopfian. However, it is not immediately clear whether $\Gamma_{n}$ admits a non-zero multiplicative invariant, or whether direct products of such groups are finitely co-Hopfian.

\section{References}

1. G. Baumslag, E. Dyer and A. Heller, The topology of discrete groups. J. Pure Appl. Algebra. 16 (1980), 1-47.

2. A. Borel, Compact Clifford-Klein forms of symmetric spaces. Topology 2 (1963), 111-122.

3. A. Borel, On the automorphisms of certain subgroups of semisimple Lie groups. In Algebraic Geometry (Papers presented at the Bombay Colloquium, 1968), Oxford University Press (Oxford, 1969), 43-73.

4. K. S. Brown, Cohomology of Groups (Graduate Texts in Mathematics 87), Springer (Berlin, 1982).

5. D. H. Gottlieb, A certain subgroup of the fundamental group. Amer. J. Math. 87 (1965), 840-856.

6. P. J. Higgins, Categories and Groupoids, Van Nostrand (Princeton, NJ, 1971).

7. F. E. A. Johnson, Linear properties of poly-Fuchsian groups. Collec. Math. 45 (1994), 183-203.

8. F. E. A. Johnson, Quasi-rigid manifolds. Preprint, University College, London, 2007.

9. G. A. Margulis, Discrete Subgroups of Lie Groups (Ergebnisse der Mathematik (Series 3) 17), Springer (Berlin, 1989).

10. G. D. Mostow, Strong Rigidity of Locally Symmetric Spaces (Annals of Mathematik Studies 78), Princeton University Press (Princeton, NJ, 1973).

11. M. S. Raghunathan, Discrete Subgroups of Lie Groups (Ergebnisse der Mathematik 68), Springer (Berlin, 1972).

12. S. Rosset, A vanishing theorm for Euler characteristics. Math. Z. 185 (1984), 211-215.

13. J. P. Serre, Cohomologie des groupes discrets. In Prospects in Mathematics (Annals of Mathematics Studies 70), Princeton University Press (Princeton, NJ, 1971), 77-169.

14. R. Strebel, A note on subgroups of infinite index in Poincaré Duality groups. Comment. Math. Helv. 52 (1977), 317-324.

15. C. T. C. Wall, Rational Euler characteristics. Proc. Cambridge Philos Soc. 57 (1961), 182-183.

J. J. A. M. Humphreys, Department of Mathematics,

University College London,

Gower Street, London WC1E 6BT,

U.K.

E-mail: jodie.humphreys@cantab.net

F. E. A. Johnson,

Department of Mathematics,

University College London,

Gower Street, London WC1E 6BT,

U.K.

E-mail: feaj@math.ucl.ac.uk
MSC (2000): Primary, 20E34, $20 \mathrm{E} 99$.

Received on the 28th of November, 2008. Accepted on the 1st of February, 2009. 